\title{
Effectiveness of computer-mediated interventions for informal carers of people with dementia - a systematic review
}

\author{
Vicky McKechnie, Chris Barker and Josh Stott \\ Research Department of Clinical, Educational and Health Psychology, University College London, London, UK
}

Background: Caring for a friend or relative with dementia can be burdensome and stressful, and puts carers at increased risk of physical and psychological problems. A number of psychosocial interventions, some delivered by computer, have been developed to support carers. This review evaluates the outcomes of computermediated interventions.

Methods: PsychINFO, MEDLINE, and CINAHL Plus were searched for papers published between January 2000 and September 2012. Study quality was evaluated using a modified version of Downs and Black's (1998) checklist.

Results: Fourteen empirical studies, evaluating a range of complex, multifaceted interventions, met inclusion criteria. The most commonly measured variables were carer burden/stress and depression. In general, higher quality studies found that interventions did have an effect on these variables. Two higher quality studies also found that anxiety was reduced following intervention. Most studies found that positive aspects of caring were increased through these interventions, as was carer self-efficacy. There were mixed results in relation to social support, and physical aspects of caring did not seem to be affected. Program impact measures indicated general acceptability of these interventions.

Conclusions: The findings support the provision of computer-mediated interventions for carers of people with dementia. Future studies would benefit from design improvements, such as articulating clearly defined aims, having a control group, having adequate statistical power, and measuring a greater range of factors important to carers themselves.

Key words: Dementia, Alzheimer's disease, social support, carers

\section{Introduction}

Many of the 750,000 people in the United Kingdom with dementia are cared at home by a relative or friend (Alzheimer's Society, 2011). Carers of people with dementia (PwD) frequently report feelings of social isolation and inadequate social support (Stoltz et al., 2004) and the risk of anxiety and depressive disorders is increased (Schulz and Martire, 2004). Furthermore, these negative outcomes are correlated with the level of cognitive impairment as well as behavioral disturbance in the person with dementia (Schoenmakers et al., 2010). Contextual factors are also important in experiences of social isolation, with lower socioeconomic status and poorer formal support for the person with

\footnotetext{
Correspondence should be addressed to: Dr. Vicky McKechnie, Research Department of Clinical, Educational and Health Psychology, University College London, 1-19 Gower Street, London, WC1E 6BT, UK. Phone: + 0207-6791897; Fax: +020-7916-1989. Email: vicky.mckechnie.10@ucl.ac.uk. Received 30 Sep 2013; revision requested 5 Feb 2014; revised version received 1 May 2014; accepted 6 May 2014. First published online 3 July 2014.
}

dementia related to greater social isolation (Robison et al., 2009). Older carers' physical health (Schulz and Martire, 2004) and mortality (Schulz and Beach, 1999) can also be affected, which can in turn compromise their ability to care for the person with dementia.

A range of interventions for carers of PwD exist to mitigate these problems (Sörensen et al., 2002). The present review is concerned with psychosocial information and communication technology (ICT) interventions aimed at providing information and improving the carer's wellbeing and coping skills (e.g., psychoeducational interventions and support groups) rather than interventions aimed at reducing the objective amount of care provided by carers (e.g., interventions that improve the patient's competence in daily activities).

Uptake of traditional dementia services, both for PwD and carers, has been low; furthermore, carers of PwD show great interest in support services other than traditional face-to-face support groups, 
for example, telephone support, newsletters, and interventions delivered on computer (Colantonio et al., 2001). Such nontraditional interventions can be similar in content to traditional interventions, but vary in their format. For example, face-toface educational sessions can also be delivered on video or DVD (see, e.g., Gant et al., 2007). Nontraditional media also open up a range of possibilities for carer support. For example, internet support groups can help individuals physically distanced from one another, 24 hours a day and seven days a week. This is particularly helpful for individuals who are physically isolated or experiencing rare or stigmatized conditions (White and Dorman, 2001). The current review will focus on one form of nontraditional interventioncomputer-mediated psychosocial interventions.

\section{Previous reviews}

There have been three previous reviews of this area. Powell et al. (2008) conducted a systematic review of networked technologies supporting informal carers of PwD. They identified 15 papers that described five different multifaceted interventions. They found that the interventions had inconsistent outcomes, but suggested that they had moderate effects on improving carer stress and depression. Treatment effects varied with carer characteristics such as ethnic group, formal support, and baseline burden. This review was published as a brief descriptive report, and its evaluation of studies was therefore limited. In addition, with the rapid development of the internet and other computer technologies, it now requires updating.

A more recent French review (Wu et al., 2009) looked at studies of information and communication technology interventions supporting carers of PwD. Sixteen papers concerning nine intervention programs (internet and telephone interventions) were described, without a critical analysis or comparison of studies, and with limited conclusions drawn.

Godwin et al. (2013) conducted a systematic review with the aim of ascertaining the psychosocial effects of technology-driven interventions for informal carers of PwD. Eight papers were evaluated. This review only included RCTs and excluded interventions that only used CDs. All the studies reviewed showed positive outcomes, but there was considerable variability in the content and delivery of interventions, as well as inconsistency in measurement and variability of outcome.

\section{Aims of this review}

Computer-mediated intervention for carers of $\mathrm{PwD}$ is a growing area and a wide range of interventions have been developed. In order for future interventions to be maximally effective, and to ensure that service providers are aware of the strengths and weaknesses of different interventions, it is important that the current evidence base be evaluated. The present review therefore aims to address the question: How effective are computermediated psychosocial interventions for informal carers of PwD?

\section{Method}

\section{Inclusion and exclusion criteria}

The inclusion criteria were:

1. The intervention must be computer mediated. This could include DVDs, CD-ROMs, the internet, or computer programs, but excludes interventions that exclusively use the telephone. The intervention does not need to exclusively be computer mediated, but that must be its main component.

2. Interventions must be psychosocial interventions, including therapy, professional, or peer support programs, educational or information programs.

3. The carers must be informal, that is, they must not be paid carers such as nursing home staff. However, the PwD can be resident either at home or in an institution.

4. Studies must include dementia as the diagnosis of the person being cared for, but do not have to be limited to dementia. All types and all stages of dementia were included.

5. Studies must use at least one quantitative measure to assess the outcome of the intervention. These measures do not need to be standardized, and can be questionnaires asking for users' views of the effects of the intervention. Studies using only questionnaires that consider features of the intervention, such as its convenience, userfriendliness, or accessibility, were excluded.

6. Studies can be randomized-controlled trials, preand posttest studies with or without a control group, or posttest-only studies with or without a control group. Case studies and qualitative research were excluded.

7. Studies must be published in peer reviewed journal articles, in English, from January 2000 to September 2012. The lower limit was chosen because the review focuses on current technologies.

\section{Search strategy}

Search terms were organized into four conceptual areas:

computer* OR DVD* OR internet* OR network* OR technolog* OR ICT OR online* OR CD-ROM* 


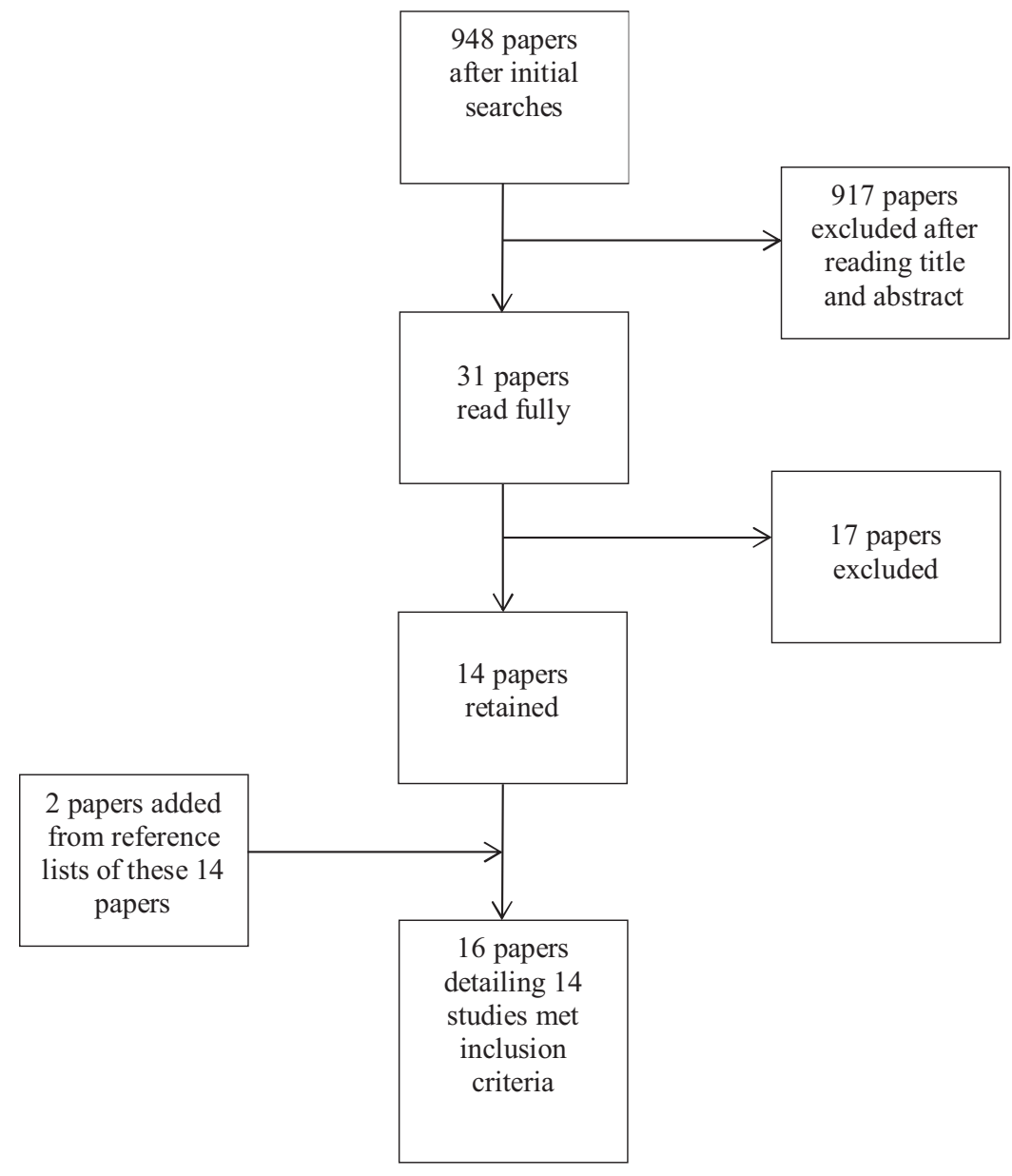

Figure 1. Flow diagram of studies included and excluded.

intervention* OR train* OR therap* OR support* OR treatment*

\section{AND}

carer* OR caring * OR caregiv* OR family* OR families* OR parent* OR mother* OR father*

\section{AND}

dement* OR Alzheimer*

Grey literature was not searched. Searches of PsychINFO, MEDLINE, and CINAHL Plus databases generated 262,708 , and 248 papers, respectively. After de-duplication, there were 948 papers of which the titles and abstracts were examined, out of which 31 papers seemed that they might potentially fulfill the inclusion criteria; the full texts of these were then read in full by the first and third authors (see Figure 1 for PRISMA flowchart).

Seventeen of these 31 papers were excluded. Seven were excluded because the intervention was not computer mediated, four because the evaluation of the intervention was limited to anecdotal reports, qualitative interviews, and/or usage or intervention acceptability information, one was a literature review, one was a description of an intervention, one was a research proposal, one was a study of the relative importance of intervention elements such as social support, one was a meta-analysis, and one investigated whether older carers were able to complete standardized questionnaires. There were queries about four papers' eligibility, and in these instances, the second author was consulted in order to reach consensus.

This left 14 papers for review. In two instances, two papers reported results from the same study (Glueckauf and Loomis, 2003; Glueckauf et al., 2004; Chiu et al., 2009; Chiu and Eysenbach, 2010). So, at this point, 12 studies were identified for review. For clarity, when these studies are referred to, Chiu et al. (2009) and Glueckauf et al. (2004) will be cited.

Reference lists from the included papers, as well as from Powell et al. (2008) and Wu et al. (2009), were examined to see whether any additional papers were eligible. This led to four further papers being examined, yielding two additional papers (Magnusson et al., 2005; Marziali and Donahue, 2006). The reference lists of these papers were 
also searched, leading to one further paper being examined, but no further studies identified. Sixteen papers, representing 14 empirical studies were evaluated.

\section{Study quality}

The quality of studies was evaluated using a modified version of Downs and Black's (1998) checklist for the assessment of the methodological quality both of randomized and nonrandomized studies of health care interventions. In light of the nature of the studies being evaluated, several items on the checklist were not relevant (items 8, 13, 14, and 19), and were therefore excluded from the assessment. For example, item 14 asked whether any attempt was made to blind study subjects to the interventions that they received, which is not relevant for most psychological intervention studies. Each study could achieve a score of between 0 and 24, with higher scores indicating better quality. Scores of 0-8 were considered to indicate studies of low quality; $9-16$ as medium quality, and $17-24$ as high quality.

\section{Results}

Table 1 summarizes the 14 studies evaluated. Several used mixed methods; the present review only addresses their quantitative components.

\section{Interventions}

The interventions were varied; many had multiple components. In nine studies, with quality scores ranging from 5 to 20 , the intervention was either primarily or entirely delivered as an internet program or resource. One high quality study used a computer-mediated automated interactive voice response intervention; two, of high and medium quality, used a computer-telephone integration system; one of high quality used a DVD program; and one low quality study used computer-, but not internet-, based education. In eight studies, the main part of the intervention was education; three combined education with professional therapy or support; two combined education with more general support; one compared education and professional support with education and more general peer support.

Their aims tended to be to reduce carer distress and increase competence in caregiving. One aimed to enhance family participation in nursing home care (Rosen et al., 2003). Five studies were labeled as pilot or feasibility studies (Rosen et al., 2003; Marziali and Donahue, 2006; Marziali and Garcia, 2006; Torp et al., 2008; van der Roest et al.,
2010) and some that were not described as pilots nonetheless involved the development of a new intervention (e.g., Glueckauf et al., 2004; Chiu et al., 2009).

Six papers described interventions that included the ongoing involvement of a professional, six described interventions that did not, with one paper comparing an intervention including a professional with one that did not. Magnusson et al. (2005) was unclear about the level of professional involvement. In only one study did it seem that the intervention was continued after it was evaluated (Torp et al., 2008). This is surprising, given that a benefit of computer-mediated interventions, particularly internet interventions, is that they can be used on an ongoing basis. Individual interventions varied in terms of the amount and frequency of input over the time period that they were offered. In most cases, carers knew the diagnosis of the care recipient but studies were not excluded if they did not.

\section{Study designs}

Six studies were randomized controlled trials, one was a controlled trial, four used a single group pretest posttest design, two used a single group posttest-only design, and one used a two group pretest posttest design. Initial sample sizes ranged from 18 (Rosen et al., 2003) to 329 (Beauchamp et al., 2005), attrition rates ranged from $0 \%$ (Rosen et al., 2003; Marziali and Garcia, 2011) to $42 \%$ (Marziali and Donahue, 2006), although several studies did not report them. Although a number of papers compared the characteristics (such as age and baseline depression score) of those who dropped out with those who did not, only one presented an intent-to-treat analysis (Chiu et al., 2009) and one interpolated 18 months follow-up scores where data were available (Eisdorfer et al., 2003).

No study provided a power calculation, and several authors noted that their study was likely to be underpowered. Several small studies used mixedmethods designs (e.g., Magnusson et al., 2005; Torp et al., 2008). Reporting of follow-up periods was often not present or was ambiguous, raising questions as to long-term impact; only Mahoney et al. (2003) and Eisdorfer et al. (2003) described follow-up periods that extended beyond the end of the intervention.

\section{Measures}

Most studies used multiple outcome measures, which is important in order to capture the range of possible impacts on carers. In several, however, multiple measures were used but only certain 
Table 1. Study characteristics

\begin{tabular}{|c|c|c|c|c|c|c|c|c|}
\hline $\begin{array}{l}\text { AUTHORS } \\
\text { AND } \\
\text { DATE }\end{array}$ & PARTICIPANTS & $\begin{array}{l}\text { INITIAL } \\
\text { SAMPLE } \\
\text { SIZE }^{*}\end{array}$ & INTERVENTION & $\begin{array}{l}\text { AIMS OF } \\
\text { STUDY }\end{array}$ & MEASURES & DESIGN & $\begin{array}{l}\text { PRINCIPAL } \\
\text { FINDINGS }\end{array}$ & $\begin{array}{l}\text { QUALITY } \\
\text { SCORE }^{\dagger}\end{array}$ \\
\hline $\begin{array}{l}\text { Beauchamp } \\
\text { et al. } \\
(2005)\end{array}$ & $\begin{array}{l}\text { Employed family } \\
\text { carers in USA }\end{array}$ & 329 & $\begin{array}{l}\text { Worksite-based } \\
\text { internet multimedia } \\
\text { program: } \\
\text { "Caregiver's Friend: } \\
\text { Dealing with } \\
\text { Dementia." } \\
\text { Program provided } \\
\text { text materials and } \\
\text { videos that modeled } \\
\text { positive caregiving } \\
\text { strategies. } 30 \text { days } \\
\text { exposure } \\
\text { (Control: wait list) }\end{array}$ & $\begin{array}{l}\text { Can exposure to } \\
\text { the program: } \\
\text { (a) improve carer } \\
\text { appraisals?; } \\
\text { (b) increase the } \\
\text { use of } \\
\text { constructive } \\
\text { coping skills?; } \\
\text { (c) reduce the } \\
\text { negative } \\
\text { outcomes of } \\
\text { depression, } \\
\text { anxiety and } \\
\text { strain?; } \\
\text { (d) increase } \\
\text { positive } \\
\text { perceptions of } \\
\text { caregiving? }\end{array}$ & $\begin{array}{l}\text { Items used } \\
\text { from: } \\
\text { CES-D; } \\
\text { STAI; } \\
\text { CSI; } \\
\text { PAC; } \\
\text { RWC }\end{array}$ & $\begin{array}{l}\text { Pretest-posttest } \\
\text { RCT; } \\
\text { Follow up at end } \\
\text { of intervention }\end{array}$ & $\begin{array}{l}\text { Treatment group } \\
\text { reported } \\
\text { greater gains } \\
\text { with respect to } \\
\text { measures of } \\
\text { self-efficacy, } \\
\text { intention to get } \\
\text { support and } \\
\text { caregiver gain, } \\
\text { and reductions } \\
\text { in carer stress, } \\
\text { strain, } \\
\text { depressive } \\
\text { symptomato- } \\
\text { logy, and state } \\
\text { anxiety }\end{array}$ & 20 \\
\hline $\begin{array}{l}\text { Chiu et al. } \\
\text { (2009) }\end{array}$ & $\begin{array}{l}\text { Chinese } \\
\text { Canadian } \\
\text { family carers of } \\
\text { elderly people } \\
\text { with } \\
\text { Alzheimer's } \\
\text { disease and } \\
\text { related } \\
\text { dementias }\end{array}$ & 35 & $\begin{array}{l}\text { Internet-based } \\
\text { Caregiver Support } \\
\text { Service (ICSS): An } \\
\text { online carer } \\
\text { information } \\
\text { handbook, and } \\
\text { exchange of e-mails } \\
\text { between caregivers } \\
\text { and Chinese } \\
\text { professional } \\
\text { clinicians } \\
\text { (occupational } \\
\text { therapists) } \\
6 \text { months access }\end{array}$ & $\begin{array}{l}\text { To explore usage } \\
\text { behavior } \\
\text { associated with } \\
\text { outcomes and } \\
\text { to evaluate } \\
\text { effects of } \\
\text { participation in } \\
\text { ICSS on carer } \\
\text { health } \\
\text { outcomes }\end{array}$ & BSFC & $\begin{array}{l}\text { Single group } \\
\text { pretest-posttest } \\
\text { Follow up at } \\
\text { end of } \\
\text { intervention }\end{array}$ & $\begin{array}{l}\text { Nonusers had an } \\
\text { increase in } \\
\text { perceived } \\
\text { burden postint- } \\
\text { ervention, } \\
\text { while frequent } \\
\text { users had a } \\
\text { decrease of } \\
\text { burden score }\end{array}$ & 12 \\
\hline
\end{tabular}


Table 1. Continued

\begin{tabular}{|c|c|c|c|c|c|c|c|c|}
\hline $\begin{array}{l}\text { AUTHORS } \\
\text { AND } \\
\text { DATE }\end{array}$ & PARTICIPANTS & $\begin{array}{l}\text { INITIAL } \\
\text { SAMPLE } \\
\text { SIZE }^{*}\end{array}$ & INTERVENTION & $\begin{array}{l}\text { AIMS OF } \\
\text { STUDY }\end{array}$ & MEASURES & DESIGN & $\begin{array}{l}\text { PRINCIPAL } \\
\text { FINDINGS }\end{array}$ & $\begin{array}{l}\text { QUALITY } \\
\text { SCORE }^{\dagger}\end{array}$ \\
\hline $\begin{array}{l}\text { Eisdorfer } \\
\text { et al. } \\
\quad(2003)\end{array}$ & $\begin{array}{l}\text { Cuban American } \\
\text { and White } \\
\text { American } \\
\text { family carers of } \\
\text { people with } \\
\text { Alzheimer's } \\
\text { Disease and } \\
\text { related } \\
\text { dementias }\end{array}$ & 225 & $\begin{array}{l}\text { Part of the REACH } \\
\text { (Resources for } \\
\text { Enhancing Alzheimer's } \\
\text { Caregiver Health) } \\
\text { program. } \\
3 \text { conditions: } \\
\text { (a) structural ecosystems } \\
\text { therapy } \\
\text { (b) structural ecosystems } \\
\text { therapy }+ \\
\text { computer-telephone } \\
\text { integration system } \\
\text { (c) minimal support } \\
\text { condition } \\
12 \text { month interventions. }\end{array}$ & $\begin{array}{l}\text { To examine the } \\
\text { efficacy of the } \\
\text { two } \\
\text { interventions } \\
\text { across Cuban } \\
\text { American and } \\
\text { White } \\
\text { American } \\
\text { carers and to } \\
\text { examine the } \\
\text { efficacy of the } \\
\text { interventions } \\
\text { over time. } \\
\text { To explore the } \\
\text { differential } \\
\text { effects of the } \\
\text { treatment } \\
\text { across varying } \\
\text { carer-care } \\
\text { recipient } \\
\text { dyads }\end{array}$ & CES-D & $\begin{array}{l}\text { Pretest-posttest } \\
\text { RCT; } \\
\text { measures at } \\
\text { baseline, } 6,12, \\
\text { and } 18 \text { months }\end{array}$ & $\begin{array}{l}\text { Carers in } \\
\text { condition (b) } \\
\text { experienced } \\
\text { reduction in } \\
\text { depressive } \\
\text { symptoms at } 6 \\
\text { months relative } \\
\text { to other } \\
\text { conditions. } \\
\text { 18-month } \\
\text { follow up } \\
\text { indicated the } \\
\text { intervention } \\
\text { was particularly } \\
\text { beneficial for } \\
\text { Cuban } \\
\text { American } \\
\text { husband and } \\
\text { daughter } \\
\text { carers. }\end{array}$ & 18 \\
\hline $\begin{array}{l}\text { Finkel } \\
\text { et al. } \\
\text { (2007) }\end{array}$ & $\begin{array}{l}\text { Family carers of } \\
\text { PwD }\end{array}$ & 46 & $\begin{array}{l}\text { Provision of information } \\
\text { about dementia and } \\
\text { community resources } \\
\text { and strategies to } \\
\text { enhance safety, } \\
\text { communication, } \\
\text { self-care, social } \\
\text { support, and } \\
\text { management of } \\
\text { problem } \\
\text { behaviors-delivered } \\
\text { through the customized } \\
\text { Computer-Telephone } \\
\text { Integration System } \\
6 \text { month intervention } \\
\text { (Control: information } \\
\text { only) }\end{array}$ & $\begin{array}{l}\text { Evaluate } \\
\text { intervention } \\
\text { effectiveness }\end{array}$ & $\begin{array}{l}\text { CES-D; } \\
\text { three } \\
\text { questions } \\
\text { assessing } \\
\text { the } \\
\text { primary } \\
\text { domains } \\
\text { of the } \\
\text { RMBPC; } \\
\text { modified } \\
\text { version of } \\
\text { CHHBS; } \\
\text { a Received } \\
\text { Social } \\
\text { Support } \\
\text { Scale from } \\
\text { the ISSB }\end{array}$ & $\begin{array}{l}\text { Pretest-posttest } \\
\text { RCT; } \\
6 \text { months follow } \\
\text { up }\end{array}$ & $\begin{array}{l}\text { Carers in } \\
\text { intervention } \\
\text { condition } \\
\text { reported a } \\
\text { decrease in } \\
\text { burden post } \\
\text { intervention, } \\
\text { and those who } \\
\text { evidenced high } \\
\text { depression at } \\
\text { baseline } \\
\text { experienced a } \\
\text { decline in } \\
\text { depression }\end{array}$ & 13 \\
\hline
\end{tabular}


Table 1. Continued

\begin{tabular}{|c|c|c|c|c|c|c|c|c|}
\hline $\begin{array}{l}\text { AUTHORS } \\
\text { AND } \\
\text { DATE }\end{array}$ & PARTICIPANTS & $\begin{array}{l}\text { INITIAL } \\
\text { SAMPLE } \\
\text { SIZE }^{*}\end{array}$ & INTERVENTION & $\begin{array}{l}\text { AIMS OF } \\
\text { STUDY }\end{array}$ & MEASURES & DESIGN & $\begin{array}{l}\text { PRINCIPAL } \\
\text { FINDINGS }\end{array}$ & $\begin{array}{l}\text { QUALITY } \\
\text { SCORE }^{\dagger}\end{array}$ \\
\hline $\begin{array}{l}\text { Gallagher- } \\
\text { Thompson } \\
\text { et al. } \\
(2010)\end{array}$ & $\begin{array}{l}\text { Chinese } \\
\text { American } \\
\text { carers living in } \\
\text { the San } \\
\text { Francisco Bay } \\
\text { area }\end{array}$ & 70 & $\begin{array}{l}\text { CBT skill training } \\
\text { program delivered on a } \\
\text { DVD (plus workbook) } \\
\text { 12-16 week } \\
\text { intervention. } \\
\text { (Control: general } \\
\text { educational DVD } \\
\text { program on dementia, } \\
\text { plus written materials } \\
\text { to supplement) }\end{array}$ & $\begin{array}{l}\text { Hypothesis- } \\
\text { that the skill } \\
\text { training DVD } \\
\text { treatment } \\
\text { would be } \\
\text { more effective } \\
\text { than the } \\
\text { educational } \\
\text { DVD in } \\
\text { reducing } \\
\text { conditional } \\
\text { bother, } \\
\text { increasing } \\
\text { positive affect, } \\
\text { and reducing } \\
\text { other negative } \\
\text { symptoms of } \\
\text { depression }\end{array}$ & $\begin{array}{l}\text { CES-D; } \\
\text { RMBPC; } \\
\text { Program } \\
\text { evaluation } \\
\text { question- } \\
\text { naire }\end{array}$ & $\begin{array}{l}\text { Pretest-posttest } \\
\text { RCT; } \\
\text { follow up } 16 \\
\text { weeks after } \\
\text { baseline }\end{array}$ & $\begin{array}{l}\text { The two } \\
\text { interventions } \\
\text { did not } \\
\text { differentially } \\
\text { affect level of } \\
\text { depressive } \\
\text { symptoms; } \\
\text { positive affect } \\
\text { subscale score } \\
\text { of CES-D } \\
\text { increased more } \\
\text { for those in skill } \\
\text { DVD group. } \\
\text { Reaction to } \\
\text { problems } \\
\text { decreased in } \\
\text { skill DVD } \\
\text { group. Both } \\
\text { groups reported } \\
\text { the intervention } \\
\text { improved their } \\
\text { confidence and } \\
\text { skills in caring, } \\
\text { with the skill } \\
\text { DVD group } \\
\text { generally } \\
\text { reporting } \\
\text { greater gains. }\end{array}$ & 18 \\
\hline
\end{tabular}


Table 1. Continued

\begin{tabular}{|c|c|c|c|c|c|c|c|c|}
\hline $\begin{array}{l}\text { AUTHORS } \\
\text { AND } \\
\text { DATE }\end{array}$ & PARTICIPANTS & $\begin{array}{l}\text { INITIAL } \\
\text { SAMPLE } \\
\text { SIZE }\end{array}$ & INTERVENTION & $\begin{array}{l}\text { AIMS OF } \\
\text { STUDY }\end{array}$ & MEASURES & DESIGN & $\begin{array}{l}\text { PRINCIPAL } \\
\text { FINDINGS }\end{array}$ & $\begin{array}{l}\text { QUALITY } \\
\text { SCORE }^{\dagger}\end{array}$ \\
\hline $\begin{array}{l}\text { Glueckauf } \\
\text { et al. } \\
\quad(2004)\end{array}$ & $\begin{array}{l}\text { Carers of } \\
\text { individuals with } \\
\text { progressive } \\
\text { dementia from } \\
\text { Maryland, } \\
\text { Florida (bar } \\
\text { one) }\end{array}$ & 40 & $\begin{array}{l}\text { Alzheimer's Caregiver } \\
\text { Support Online } \\
\text { (AlzOnline)-an } \\
\text { internet- and } \\
\text { telephone-based } \\
\text { education and support } \\
\text { network for carer of } \\
\text { individuals with } \\
\text { progressive dementia } \\
16 \text { week intervention } \\
\text { on average }\end{array}$ & $\begin{array}{l}\text { Initial program } \\
\text { evaluation of } \\
\text { AlzOnline's } \\
\text { Positive } \\
\text { Caregiving } \\
\text { Classes. } \\
\text { Objectives: To } \\
\text { assess the } \\
\text { impact of the } \\
\text { program on } \\
\text { the } \\
\text { psychosocial } \\
\text { functioning of } \\
\text { carer } \\
\text { participants, } \\
\text { particularly } \\
\text { their } \\
\text { perceptions of } \\
\text { self-efficacy, } \\
\text { emotional } \\
\text { growth, and } \\
\text { burden from } \\
\text { the caregiving } \\
\text { experience }\end{array}$ & $\begin{array}{l}\text { CSES; } \\
\text { SRGS; } \\
\text { CAI }\end{array}$ & $\begin{array}{l}\text { Single group } \\
\text { pretest-posttest }\end{array}$ & $\begin{array}{l}\text { Participants } \\
\text { reported pre- to } \\
\text { postclass } \\
\text { increases on all } \\
3 \text { CSES } \\
\text { subscales, and } \\
\text { concomitant } \\
\text { decreases in } \\
\text { subjective carer } \\
\text { burden. There } \\
\text { were } \\
\text { substantial } \\
\text { improvements } \\
\text { in their } \\
\text { perceptions of } \\
\text { self-efficacy in } \\
\text { performing } \\
\text { routine } \\
\text { caregiving } \\
\text { duties and } \\
\text { managing } \\
\text { challenging } \\
\text { care recipient } \\
\text { behaviors, and } \\
\text { their appraisals } \\
\text { of the } \\
\text { emotional } \\
\text { caregiver } \\
\text { burden from } \\
\text { pre- to } \\
\text { posttesting } \\
\text { phase }\end{array}$ & 10 \\
\hline
\end{tabular}


Table 1. Continued

\begin{tabular}{|c|c|c|c|c|c|c|c|c|}
\hline $\begin{array}{l}\text { AUTHORS } \\
\text { AND } \\
\text { DATE }\end{array}$ & PARTICIPANTS & $\begin{array}{l}\text { INITIAL } \\
\text { SAMPLE } \\
\text { SIZE* }\end{array}$ & INTERVENTION & $\begin{array}{l}\text { AIMS OF } \\
\text { STUDY }\end{array}$ & MEASURES & DESIGN & $\begin{array}{l}\text { PRINCIPAL } \\
\text { FINDINGS }\end{array}$ & $\begin{array}{l}\text { QUALITY } \\
\text { SCORE }^{\dagger}\end{array}$ \\
\hline $\begin{array}{l}\text { Lewis et al. } \\
\quad(2010)\end{array}$ & $\begin{array}{l}\text { Carers of people } \\
\text { with dementia } \\
\text { in USA }\end{array}$ & 63 & $\begin{array}{l}\text { Internet-Based Savvy } \\
\text { Caregiver (ISBC)-an } \\
\text { internet-based } \\
\text { psychoeducational } \\
\text { program }\end{array}$ & $\begin{array}{l}\text { To establish } \\
\text { feasibility and } \\
\text { acceptability } \\
\text { of program }\end{array}$ & $\begin{array}{l}\text { Follow-up } \\
\text { question- } \\
\text { naire }\end{array}$ & $\begin{array}{l}\text { Single group } \\
\text { posttest }\end{array}$ & $\begin{array}{l}\text { Participants } \\
\text { found the } \\
\text { program } \\
\text { educational, } \\
\text { convenient, } \\
\text { useful, and } \\
\text { interesting. } \\
\text { They endorsed } \\
\text { feeling more } \\
\text { confident in } \\
\text { caring skills } \\
\text { and } \\
\text { communication } \\
\text { with their } \\
\text { family. }\end{array}$ & 9 \\
\hline $\begin{array}{l}\text { Magnusson } \\
\text { et al. } \\
\text { (2005) }\end{array}$ & $\begin{array}{l}\text { Family carers of } \\
\text { older people in } \\
\text { two } \\
\text { municipalities } \\
\text { in the west of } \\
\text { Sweden. } \\
\text { Carers: } 16 \\
\text { stroke, } 5 \\
\text { dementia, } 5 \\
\text { diabetes, and } \\
\text { various other }\end{array}$ & 34 & $\begin{array}{l}\text { Swedish ACTION } \\
\text { (Assisting family } \\
\text { Carers using } \\
\text { Telematics } \\
\text { Interventions to meet } \\
\text { Older persons' Needs) } \\
\text { project: An ICT service } \\
\text { providing carers with } \\
\text { information, } \\
\text { education, and support } \\
\text { Intervention ranged from } \\
3 \text { months to } 1 \text { year. }\end{array}$ & $\begin{array}{l}\text { Program } \\
\text { evaluation }\end{array}$ & $\begin{array}{l}\text { Modified } \\
\text { version of } \\
\text { the PREP } \\
\text { evaluation } \\
\text { question- } \\
\text { naire }\end{array}$ & $\begin{array}{l}\text { Single group } \\
\text { posttest }\end{array}$ & $\begin{array}{l}\text { The intervention } \\
\text { had a } \\
\text { moderately } \\
\text { positive effect } \\
\text { on the } \\
\text { preparedness, } \\
\text { rewards, and } \\
\text { satisfaction of } \\
\text { caring }\end{array}$ & 5 \\
\hline
\end{tabular}




\begin{tabular}{|c|c|c|c|c|c|c|c|c|}
\hline $\begin{array}{l}\text { AUTHOF } \\
\text { AND } \\
\text { DATE }\end{array}$ & PARTICIPANTS & $\begin{array}{l}\text { INITIAL } \\
\text { SAMPLE } \\
\text { SIZE* }^{*}\end{array}$ & INTERVENTION & $\begin{array}{l}\text { AIMS OF } \\
\text { STUDY }\end{array}$ & MEASURES & DESIGN & $\begin{array}{l}\text { PRINCIPAL } \\
\text { FINDINGS }\end{array}$ & $\begin{array}{l}\text { QUALITY } \\
\text { SCORE }^{\dagger}\end{array}$ \\
\hline $\begin{array}{l}\text { Mahoney } \\
\text { et al. } \\
\quad(2003)\end{array}$ & $\begin{array}{l}\text { Family carers of } \\
\text { people with } \\
\text { Alzheimer's } \\
\text { Disease in USA }\end{array}$ & 100 & $\begin{array}{l}\text { Part of the REACH } \\
\text { (Resources for } \\
\text { Enhancing Alzheimer's } \\
\text { Caregiver Health) } \\
\text { program. Technology } \\
\text { intervention-access to } \\
\text { a computer-mediated } \\
\text { automated interactive } \\
\text { voice response (IVR) } \\
\text { intervention } \\
12 \text { month intervention } \\
\text { (Control: usual care) }\end{array}$ & $\begin{array}{l}\text { To investigate } \\
\text { whether this } \\
\text { system could } \\
\text { reduce stress } \\
\text { associated } \\
\text { with caring for } \\
\text { a family } \\
\text { member with } \\
\text { Alzheimer's } \\
\text { related } \\
\text { disruptive } \\
\text { behaviors. }\end{array}$ & $\begin{array}{l}\text { RMBPC; } \\
\text { STAI; } \\
\text { CES-D }\end{array}$ & $\begin{array}{l}\text { Pretest- posttest } \\
\text { RCT; } \\
\text { measures at } \\
\text { baseline, } 6,12, \\
\text { and } 18 \text { months }\end{array}$ & $\begin{array}{l}\text { Participants with } \\
\text { lower mastery } \\
\text { at baseline } \\
\text { showed } \\
\text { improvement in } \\
\text { bother, anxiety } \\
\text { and depression. } \\
\text { Wives showed a } \\
\text { reduction of } \\
\text { the bothersome } \\
\text { nature of } \\
\text { caregiving }\end{array}$ & 21 \\
\hline $\begin{array}{l}\text { Marziali } \\
\text { and } \\
\text { Do- } \\
\text { nahue } \\
(2006)\end{array}$ & $\begin{array}{l}\text { Family carers in } \\
\text { Canada of older } \\
\text { adults with } \\
\text { neurodegener- } \\
\text { ative diseases } \\
\text { (Alzheimer's; } \\
\text { stroke-related } \\
\text { dementia; } \\
\text { Parkinson's) }\end{array}$ & 66 & $\begin{array}{l}\text { "Caring For Others" } \\
\text { intervention program. } \\
\text { Intervention group } \\
\text { received computers } \\
\text { and access to a website } \\
\text { with links to } \\
\text { information, e-mail } \\
\text { and threaded } \\
\text { discussion, } \\
\text { video-conferencing link } \\
\text { ( } \rightarrow 10 \text {-session } \\
\text { manual-guided } \\
\text { psychosocial support } \\
\text { group facilitated by a } \\
\text { group therapist, } \\
\text { followed by } 12 \\
\text { additional online } \\
\text { sessions facilitated by a } \\
\text { group member) } \\
22 \text { week intervention } \\
\text { (Control: no } \\
\text { intervention) }\end{array}$ & $\begin{array}{l}\text { To evaluate the } \\
\text { effects of the } \\
\text { intervention } \\
\text { on carers }\end{array}$ & $\begin{array}{l}\text { HSQ-12; } \\
\text { CES-D; } \\
\text { a measure } \\
\text { requiring } \\
\text { carers to } \\
\text { endorse } \\
\text { pres- } \\
\text { ence/absence } \\
\text { of activities } \\
\text { of daily } \\
\text { living and } \\
\text { instru- } \\
\text { mental } \\
\text { ADLs } \\
\text { performed } \\
\text { for care } \\
\text { recipient, } \\
\text { rating } \\
\text { degree of } \\
\text { stress for } \\
\text { each; } \\
\text { RMBPC; } \\
\text { MSPSS }\end{array}$ & $\begin{array}{l}\text { Pretest- posttest } \\
\text { RCT; } \\
\text { measures at } \\
\text { baseline and } \\
\text { 6-month follow } \\
\text { up }\end{array}$ & $\begin{array}{l}\text { Intervention } \\
\text { group } \\
\text { experienced a } \\
\text { decline in stress }\end{array}$ & 12 \\
\hline
\end{tabular}


Table 1. Continued

\begin{tabular}{|c|c|c|c|c|c|c|c|c|}
\hline $\begin{array}{l}\text { AUTHORS } \\
\text { AND } \\
\text { DATE }\end{array}$ & PARTICIPANTS & $\begin{array}{l}\text { INITIAL } \\
\text { SAMPLE } \\
\text { SIZE }^{*}\end{array}$ & INTERVENTION & $\begin{array}{l}\text { AIMS OF } \\
\text { STUDY }\end{array}$ & MEASURES & DESIGN & $\begin{array}{l}\text { PRINCIPAL } \\
\text { FINDINGS }\end{array}$ & $\begin{array}{l}\text { QUALITY } \\
\text { SCORE }^{\dagger}\end{array}$ \\
\hline $\begin{array}{l}\text { Marziali } \\
\text { and } \\
\text { Garcia } \\
(2011)\end{array}$ & $\begin{array}{l}\text { Canadian carers } \\
\text { (French and } \\
\text { English } \\
\text { speaking) from } \\
\text { three cities }\end{array}$ & 91 & $\begin{array}{l}\text { Two internet } \\
\text { interventions: a } \\
\text { text-based chat group } \\
\text { (including access to a } \\
\text { carer information } \\
\text { handbook and six } \\
\text { videos on managing } \\
\text { caregiving tasks); and } \\
\text { video conferencing } \\
\text { psychotherapeutic } \\
\text { support group } \\
\text { intervention facilitated } \\
\text { by a clinician, plus } \\
\text { access to a carer } \\
\text { information handbook } \\
\text { 6-month intervention } \\
\text { for chat group; } \\
\text { 20-week intervention } \\
\text { for support group. }\end{array}$ & $\begin{array}{l}\text { To examine the } \\
\text { impact on } \\
\text { dementia } \\
\text { carers' } \\
\text { experienced } \\
\text { stress and } \\
\text { health status }\end{array}$ & $\begin{array}{l}\text { HSQ-12; } \\
\text { CES-D; } \\
\text { SMAF }\end{array}$ & $\begin{array}{l}\text { Two group } \\
\text { pretest- } \\
\text { posttest, with } \\
\text { follow up at } 6 \\
\text { months }\end{array}$ & $\begin{array}{l}\text { In contrast to the } \\
\text { chat group, the } \\
\text { video group } \\
\text { showed greater } \\
\text { improvement } \\
\text { in mental } \\
\text { health status }\end{array}$ & 14 \\
\hline $\begin{array}{l}\text { Rosen et al. } \\
\quad(2003)\end{array}$ & $\begin{array}{l}\text { Family members } \\
\text { ("primary } \\
\text { decision } \\
\text { makers") of } \\
\text { nursing home } \\
\text { residents with } \\
\text { dementia living } \\
\text { in nursing } \\
\text { homes around } \\
\text { Pittsburgh, } \\
\text { Pennsylvania, } \\
\text { USA. }\end{array}$ & 18 & $\begin{array}{l}\text { Computer-based } \\
\text { education on dementia, } \\
\text { agitation/aggression, } \\
\text { and carer stress } \\
\text { 45-min intervention }\end{array}$ & $\begin{array}{l}\text { To enhance } \\
\text { family } \\
\text { participation } \\
\text { in nursing } \\
\text { home care }\end{array}$ & $\begin{array}{l}\text { Knowledge } \\
\text { question- } \\
\text { naire }\end{array}$ & $\begin{array}{l}\text { Single group } \\
\text { pretest-posttest }\end{array}$ & $\begin{array}{l}\text { Knowledge of the } \\
\text { key principles } \\
\text { of dementia } \\
\text { care improved }\end{array}$ & 8 \\
\hline
\end{tabular}


Table 1. Continued

\begin{tabular}{|c|c|c|c|c|c|c|c|c|}
\hline $\begin{array}{l}\text { AUTHORS } \\
\text { AND } \\
\text { DATE }\end{array}$ & PARTICIPANTS & $\begin{array}{l}\text { INITIAL } \\
\text { SAMPLE } \\
\text { SIZE* }\end{array}$ & INTERVENTION & $\begin{array}{l}\text { AIMS OF } \\
\text { STUDY }\end{array}$ & MEASURES & DESIGN & $\begin{array}{l}\text { PRINCIPAL } \\
\text { FINDINGS }\end{array}$ & $\begin{array}{l}\text { QUALITY } \\
\text { SCORE }^{\dagger}\end{array}$ \\
\hline $\begin{array}{l}\text { Van der } \\
\text { Roest } \\
\text { et al. } \\
\text { (2010) }\end{array}$ & $\begin{array}{l}\text { Carers of } \\
\text { community } \\
\text { dwelling PwD } \\
\text { in Amsterdam }\end{array}$ & 29 & $\begin{array}{l}\text { DEMentia-specific } \\
\text { dynamic interactive } \\
\text { social chart } \\
\text { (DEM-DISC)—a } \\
\text { demand-oriented } \\
\text { web-based social chart } \\
\text { for dementia care that } \\
\text { aims to provide users } \\
\text { with customized } \\
\text { answers about } \\
\text { potentially relevant } \\
\text { care and support } \\
\text { services in their region. } \\
\text { (Control: access to } \\
\text { pre-existing } \\
\text { information sources) }\end{array}$ & $\begin{array}{l}\text { To evaluate the } \\
\text { impact on the } \\
\text { daily life of } \\
\text { carers and the } \\
\text { usefulness of } \\
\text { the first } \\
\text { prototype of } \\
\text { DEM-DISC. }\end{array}$ & $\begin{array}{l}\text { SSCQ; } \\
\text { PMS; a } \\
\text { question- } \\
\text { naire on } \\
\text { knowledge } \\
\text { about care } \\
\text { and } \\
\text { welfare } \\
\text { services; } \\
\text { USE } \\
\text { question- } \\
\text { naire. }\end{array}$ & $\begin{array}{l}\text { Pretest posttest } \\
\text { with control } \\
\text { group (not } \\
\text { randomized); } \\
\text { follow up } 2 \\
\text { months after } \\
\text { baseline }\end{array}$ & $\begin{array}{l}\text { Following the } \\
\text { intervention, } \\
\text { carers in the } \\
\text { intervention } \\
\text { group reported } \\
\text { a higher feeling } \\
\text { of competence } \\
\text { than carers in } \\
\text { the control } \\
\text { group }\end{array}$ & 16 \\
\hline
\end{tabular}

`All findings reported in this table were statistically significant at $p<.05$, with the exception of Lewis et al. (2010) and Magnusson et al. (2005), who reported only descriptive statistics, as well as Torp et al.'s (2008) measure of forum usage, and Gallagher-Thompson et al.'s (2010) program evaluation questionnaire, which were both evaluated using descriptive statistics.

*i.e. sample size at the start of the study, before any participants dropped out.

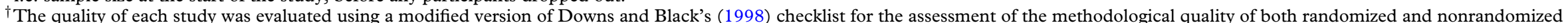
studies of healthcare interventions. Each study could achieve a score of between 0 and 24 , with higher scores indicating better quality. Scores of $0-8$ were considered to indicate studies of low quality; $9-16$ as medium quality, and $17-24$ as high quality.

BSFC = Burden Scale for Family Caregivers (Gräsel et al., 2003); CAI = Caregiver Appraisal Inventory (Lawton et al., 1989); CES-D = Center for Epidemiologic Studies-Depression scale (Radloff et al., 1977); CHHBS = Caregiver Health and Health Behaviours Scale (Posner et al., 1993); CSES = Caregiving Self-Efficacy Scale (Steffen et al., 2002); CSI = Caregiver Strain Instrument (Bass et al., 1998); FFCS = Family and Friendship Contacts Scale (Andersson, 1984); GHQ-20 = General Health Questionnaire 20 (Goldberg and Williams, 1991); HSQ-12 = Health Status Questionnaire 12 (Pettit et al., 2001); ISSB = Inventory of Socially Supportive Behaviours (Barrera et al., 1981); MSPSS = Multidimensional Scale of Perceived Social Support (Zimit et al., 1988); PAC = Positive Aspects of Caregiving (Tarlow et al., 2004); PMS = Pearlin Mastery Scale (Pearlin and Schooler, 1978); PREP evaluation questionnaire (Archbold et al.,

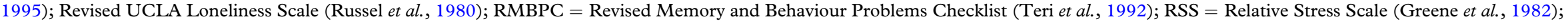
RWC = Revised Ways of Coping (Vitaliano et al., 1985); SMAF = Functional Autonomy Measurement System (Hébert et al., 2003); SRGS = Stress-related Growth Scale (Parke et al., 1996);

SSCQ = Short Sense of Competence Questionnaire (Vernooij-Dassen et al., 1999); STAI = State Trait Anxiety Inventory (Spielberger et al., 1985). 
outcomes were reported, and in some cases, only certain components of measures used were analyzed (e.g., Marziali and Donahue, 2006; Finkel et al., 2007). It was also not always made explicit why certain measures were chosen. There is a question in the wider literature as to what constitutes effectiveness, and Magnusson et al. (2005) call for meaningful, realistic outcome measures with a clearer conceptual link with the intervention aims. In some studies, this link was somewhat tenuous. For example, Rosen et al.'s (2003) intervention aimed to increase family participation in nursing home care, but assessed carers' knowledge of dementia.

\section{Outcome domains}

Studies used a wide range of carer self-report measures to evaluate interventions, and most used more than one outcome measure. The review of findings of studies is grouped according to outcomes measured, and consequently the same study may be reviewed in more than one section.

\section{MOOD AND MENTAL HEALTH}

Eight studies used measures of carer mental health, including measures of depression, anxiety, and general mental health.

Depression. Seven studies measured depression. All used variants of the Center for Epidemiologic Studies Depression Scale (CES-D; Radloff, 1977). Four high quality studies found either an improvement in CES-D score (Eisdorfer et al., 2003; Beauchamp et al. 2005), or partial effects on depression, in terms of finding an effect on a subscale, or among a subgroup of participants (Mahoney et al., 2003; Gallagher-Thompson et al., 2010). Three medium quality studies found either no main effect on depression (although post-hoc effects) (Finkel et al., 2007), no effect on depression (Marziali and Donahue, 2006), or did not report the results despite measuring depression (Marziali and Garcia, 2011).

Beauchamp et al. (2005) found a dose-response relationship showing that greater time spent viewing the internet program was associated with greater improvements on a composite outcome measure, which included depression. There are some tentative suggestions that other factors may moderate the relationship between intervention and depression. Eisdorfer et al. (2003) found that the efficacy of the intervention differed according to ethnicity and type of carer, in terms of their relationship to the person being cared for, and Finkel et al.'s (2007) post-hoc tests found that carers with greater baseline depression demonstrated greater improvements in depression.
Anxiety. Two high quality studies (Mahoney et al., 2003; Beauchamp et al., 2005) measured state anxiety using the State-Trait Anxiety Inventory (STAI; Spielberger et al., 1985). Evidence from these two studies suggests that the computermediated interventions reduced carer anxiety, although Mahoney et al. (2003) only found this result in relation to participants with medium or low levels of mastery at baseline.

General mental health. One study of Marziali and Garcia (2011) measured general mental health using the mental health subscale of the Health Status Questionnaire 12 (HSQ-12, Pettit et al., 2001). Significant results were only obtained after conducting multiple post-hoc tests without correction for family-wise error rate. Torp et al. (2008) used the General Health Questionnaire 20 (Goldberg and Williams, 1991) and did not find an effect. Beauchamp et al. (2005) used two subscales from the Revised Ways of Coping (Vitaliano et al., 1985) in their higher quality study, but did not find any differences over time between the control group and the treatment group.

\section{CARER PHYSICAL HEALTH AND HEALTH}

\section{BEHAVIORS}

Marziali and Donahue (2006) measured carer physical health using the Health Status Questionnaire 12 and Finkel et al. (2007) measured carer self-care activities using a modified version of the Caregiver Health and Health Behaviors Scale (Posner et al., 1993). Neither study reported any effects.

\section{CARER STRESS AND BURDEN}

Nine studies measured carer stress or burden. Four used variants of the Revised Memory and Behaviour Problems Checklist (RMBPC, Teri et al., 1992) (Mahoney et al., 2003; Marziali and Donahue, 2006; Finkel et al., 2007; Gallagher-Thompson et al., 2010) and the other studies used a variety of other measures. Five studies of medium and high quality found positive effects of the intervention on carer burden (Glueckauf et al., 2004; Beauchamp et al., 2005; Gallagher-Thompson et al., 2010; van der Roest et al., 2010; Marziali and Garcia, 2011). Three studies of medium and high quality found some effects of the intervention on carer burden (Mahoney et al., 2003; Marziali and Donahue, 2006; Chiu et al., 2009). Mahoney et al. (2003) found that participants with lower mastery at baseline showed greater improvements in the bothersome nature of caring, and that wives showed a decrease in the bothersome nature of caring compared to husband, child, sibling, or other relation carers. Marziali and Donahue (2006) found that the intervention group experienced a reduction 
in stress. There is some limited evidence of doseresponse relationship: Chiu et al. (2009) found that change on the Burden Scale for Family Caregivers (BSFC, Gräsel et al., 2003) was not significant overall, but that frequent users had greater change than nonusers. One study found no effect on carer burden as a result of the intervention (Torp et al., 2008).

In a high quality study, Gallagher-Thompson et al. (2010) found that mean upset or bother in relation to problems decreased in a skills learning DVD group, but not in an educational DVD group, perhaps indicating that information needs to be combined with other forms of support to reduce carer burden.

\section{CARER SOCIAL SUPPORT}

Three medium quality studies used measures of social support (Finkel et al., 2007 - A received social support scale from the Inventory of Socially Supportive Behaviours, Barrera et al., 1981; Marziali and Donahue, 2006 - The Multidimensional Scale of Perceived Social Support, Zimit et al., 1988; Torp et al., 2008 - the Revised UCLA Loneliness Scale, Russel et al., 1980, and the Family and Friendship Contacts Scale, Andersson, 1984) with varied findings.

Finkel et al. (2007) found that although there were no main effects for received social support, those in the intervention condition with higher levels of support at baseline were more likely to maintain that support than those with lower levels of support. Marziali and Donahue (2006) did not find a difference between intervention and control groups in relation to perceived social support at follow-up. Torp et al. (2008) found increases in social contacts and social support at follow-up. This is perhaps not a surprising finding given that a significant part of the intervention involved introducing computer novices to the internet, which would be likely to increase their capacity for social networking.

\section{Positive ASPECTS OF CAREgIVING}

Two studies used measures of the positive elements of caregiving (Beauchamp et al., 2005 - Positive Aspects of Caregiving, Tarlow et al., 2004); Glueckauf et al., 2004 - Stress-Related Growth Scale, Parke et al., 1996). The high quality study (Beauchamp et al., 2005) found that the intervention increased positive elements of caring, but the medium quality study (Glueckauf et al., 2004) did not.

\section{CARER SELF-EFFICACY}

One medium quality study (Glueckauf et al., 2004), found that carers reported pre to post improvements on all three subscales of the Caregiving Self-Efficacy scale (Steffen et al., 2002). Another medium quality study (van der Roest et al., 2010) did not find an effect of the intervention on carer self-efficacy. A high-quality study (Beauchamp et al., 2005) asked participants six "self-efficacy questions" and found that compared to the control group, the treatment group reported greater gains with respect to selfefficacy.

\section{PROGRAM IMPACT MEASURES AND}

\section{COMPOSITE MEASURES}

Five studies used items or measures that asked participants how the intervention had changed aspects of caring for them (Magnusson et al., 2005; Torp et al., 2008; Gallagher-Thompson et al., 2010; Lewis et al., 2010; van der Roest et al., 2010). In this review, these measures are referred to as program impact measures. One additional study used a 16item knowledge questionnaire (Rosen et al., 2003). All but one of these studies found positive effects on these measures. For the most part, the studies that used these measures were of poorer quality. This is, in part, because studies that use such measures as their only outcome measure cannot score highly on the modified version of Downs and Black's (1998) quality assessment tool.

Lewis et al. (2010) was one of two studies that only used a program impact measure. Participants generally endorsed finding the intervention to be educational, convenient, useful, and interesting. Lewis et al. (2010) generally did not find any significant relationships between demographic data and responses, apart from a low correlation between age and the caregiving scale; as age increased, scores on the caregiving scale decreased, indicating less perceived benefit from the intervention.

In Torp et al.'s (2008) study, at follow-up, carers reported less need for information about the caredfor person's illness and caring. However, whether this knowledge would have equated to an increase in ability or confidence in caring for the person cannot be established.

Rosen et al. (2003) used a 16-item knowledge questionnaire before and after a computer-based education intervention for family members of PwD who were nursing home residents. They found that knowledge of key principles of dementia care improved after the intervention. It is worth noting, however, that the mean score on the knowledge questionnaire increased by 2.3 items. While this was statistically significant, such an increase may not mean much in practice. 
Van der Roest et al. (2010) found no difference between the control group and the intervention group's knowledge about services following the intervention. They also found that the intervention group's mean opinion on the usefulness of the intervention was neutral.

\section{Discussion}

Sixteen papers describing 14 studies met inclusion criteria. Interventions were varied and multifaceted, with a range of different outcome measures. These factors make direct comparison of studies difficult, in line with previous reviews of support for carers of PwD (e.g., Wu et al., 2009). All studies found some positive effects of the intervention evaluated, although the lack of statistical power of many studies and the use of some nonstandardized measures further complicates comparison of studies.

Having said this, the most commonly measured variables were carer burden/stress and depression. In general, higher quality studies found that interventions did have an effect on these variables. Two high quality studies also found that anxiety was reduced following intervention. Positive aspects of caring may also be increased through these interventions as may through carer self-efficacy. There were mixed results in relation to social support, and physical aspects of caring did not seem to be affected. Program impact measures indicate general acceptability of these interventions.

\section{Factors influencing the effectiveness of interventions}

Due to interventions being multicomponent and complex, it is difficult to disentangle the efficacy of individual intervention components, with a large range of factors having potential effects, including the intensity and duration of the intervention, carer characteristics, and outcome selected (Cooke et al., 2001). Several of the reviewed studies attempted to examine factors influencing outcomes, although this was rarely the main focus and there was no obvious overarching framework guiding which factors to examine. Two studies found that intervention effects were moderated by certain baseline characteristics of participants, with the intervention having greater effect for those with lower mastery (Mahoney et al., 2003) or greater depression (Finkel et al., 2007). An interesting speculation is that this could indicate that the interventions are more effective for those who have more problems to start with. It could, however, also simply represent regression to the mean. As found in earlier research (e.g., George and Gwyther, 1986), two high quality studies
(Eisdorfer et al., 2003; Mahoney et al., 2003) found differential effects of interventions according carercare recipient relationship and ethnicity. These findings caution against generalizing results of research too widely and highlight the need for such factors to be considered in the design of interventions for carers of $\mathrm{PwD}$, something which is perhaps more easily incorporated in multimedia than traditionally delivered interventions. It is also potentially the case that educational information alone does not have much effect and that it needs to be combined with learning skills (GallagherThompson et al., 2010). One possible issue with computer-mediated interventions is the need for carers to be self-motivated; one high quality study (Beauchamp et al., 2005) highlighted this when they found a dose-response relationship between viewing the intervention program and change in a global composite outcome variable.

\section{Methodological issues}

The studies reviewed had a number of limitations: they often had poorly defined aims, many did not have control groups, a number were underpowered and follow-up periods were often short. Future research would benefit from addressing these issues. It is also important that outcome measures be closely linked to the aims of interventions in order that the intervention's effectiveness can be properly evaluated.

\section{Clinical implications and future research}

Computer-mediated intervention for informal carers of $\mathrm{PwD}$ is a growing area; these interventions offer a range of potential benefits. This review found that their effectiveness is mixed, but generally positive. This suggests that it would be beneficial to carers, and also to services-in terms of reaching more carers as well as potential cost saving implications-for this medium of intervention to be developed so that more individuals can benefit. As home computer use and mobile devices become increasingly widespread, the viability of computermediated interventions increases, and the cost to service providers decreases since increasing numbers of carers already have the requisite hardware. More research is, however, needed in order to ensure that interventions are maximally effective. Research needs to consider the effects of interventions on people of different ethnicities and carer-care recipient relationships, as there is evidence that differential effects exist between groups.

Although it was beyond the scope of this review to consider the qualitative components of studies, information gathered from interviews with carers 
who have used computer-mediated interventions offers an important supplement to quantitative outcome measures. It provides deeper insight into carers' experiences of using the interventions, and is able to go beyond measurement of symptom reduction and tap into factors, such as feeling supported and less alone, that are of great importance to carers. Future research into computer-mediated interventions for carers of $\mathrm{PwD}$ would benefit from a mixed-method approach.

\section{Conflict of interest}

None

\section{Description of authors' roles}

Vicky McKechnie conducted this literature review as part of her DClinPsy thesis. She located and evaluated the papers in this review, and wrote the first draft of the paper.

Joshua Stott was the joint supervisor for Vicky's DClinPsy thesis. He provided supervision throughout the project and he has commented on and edited several drafts of the paper.

Chris Barker was the joint supervisor for Vicky's DClinPsy thesis. He provided supervision throughout the project and he has commented on and edited several drafts of the paper.

\section{References}

Alzheimer's Society (2011). Alzheimer's Society: Statistics. Available at: http://www.alzheimers.org.uk/site/scripts/ documents_info.php?documentID $=341$

Andersson, L. (1984). Intervention against loneliness in a group of elderly women: a process evaluation, Human Relations, 37, 295-310.

Archbold, P. G., Steward, B. J. and Miller, L. L. (1995). The PREP system of nursing interventions: a pilot test with families caring for older members. Research in Nursing and Health, 18, 1-16.

Barrera, M., Sandler, I. and Ramsay, T. (1981). Preliminary development of a scale of social support: studies on college students. American fournal of Community Psychology, 9, 435-441.

Bass, D. M, McClendon, M. J., Flatley-Brennan, P. and McCarthy, C. (1998). The buffering effect of a computer support network on caregiver strain. Fournal of Aging and Health, 10, 20-43.

Beauchamp, N., Irvine, A. B., Seeley, J. and Johnson, B. (2005). Worksite-based internet multimedia program for family caregivers of persons with dementia. The Gerontologist, 45, 793-801.
Chiu, T. M. L. and Eysenbach, G. (2010). Stages of use: consideration, initiation, utilization, and outcomes of an internet-mediated intervention. BMC Medical Informatics and Decision Making, 10, 73-83.

Chiu, T. et al. (2009). Internet-based caregiver support for Chinese Canadians taking care for a family member with Alzheimer Disease and related dementia. Canadian fournal on Aging, 28, 323-336.

Colantonio, A., Cohen, C. and Pon, M. (2001). Assessing support needs of caregivers of persons with dementia: who wants what ? Community Mental Health fournal, 37, 231-243.

Cooke, D. D., McNally, L., Mulligan, K. T., Harrison, M. J. G and Newman, S. P. (2001). Psychosocial interventions for caregivers of people with dementia: a systematic review. Aging and Mental Health, 5, 120-135.

Downs, S. H. and Black, N. (1998). The feasibility of creating a checklist for the assessment of the methodological quality both of randomised and non-randomised studies of health care interventions. Fournal of Epidemiology and Community Health, 52, 377384.

Eisdorfer, C. et al. (2003). The effect of a family therapy and technology-based intervention on caregiver depression. The Gerontologist, 43, 521-531.

Finkel, S., Czaja, S. J., Schulz, R., Martinovick, Z., Harris, C. and Pezzuto, D. (2007). E-care: a telecommunications technology intervention for family caregivers of dementia patients. American fournal of Geriatric Psychiatry, 15, 443-448.

Gallagher-Thompson, D. et al. (2010). Effectiveness of a psychoeducational skill training DVD program to reduce stress in Chinese American dementia caregivers: results of a preliminary study. Aging and Mental Health, 14, 263-273.

Gant, J. R., Steffen, A. M. and Lauderdale, S. A. (2007). Comparative outcomes of two distance-based interventions for male caregivers of family members with dementia. American fournal of Alzheimer's Disease and Other Dementias, 22, 120-128.

George, L. K. and Gwyther, L. P. (1986). Caregiver well-being: a multidimensional examination of family caregivers of demented adults. The Gerontologist, 26, 253-259.

Glueckauf, R. L., Ketterson, T. U., Loomis, J. S. and Dages, P. (2004). Online support and education for dementia caregivers: overview, utilization, and initial program evaluation. Telemedicine fournal and e-Health, 10, 223-232.

Glueckauf, R. L. and Loomis, J. S. (2003). Alzheimer's caregiver support online: lessons learned, initial findings and future directions. NeuroRehabilitation, 18, 135-146.

Godwin, K. M., Mills, W. L., Anderson, J. A. and Kunik, M. E. (2013) Technology-driven interventions for caregivers of people with dementia: a systematic review. American Fournal of Alzheimer's Disease and Other Dementias, 28, 216-222.

Goldberg, D. and Williams, P. (1991). User's guide to the General Health Questionnaire. London: NFER-Nelson.

Gräsel, E., Chiu, T. and Oliver, R. (2003). Development and validation of the Burden Scale for Family Caregivers. Toronto, Canada: COTA Comprehensive Rehabilitation and Mental health Services. 
Greene, J. G., Smith, R., Gardiner, M. and Timbury, G. C. (1982). Measuring behavioural disturbance of elderly demented patients in the community and its effects on relatives: a factor analytic study. Age and Ageing, 11, 121-126.

Hébert, R., Durand, P. J. and Dubuc, N. (2003). The functional autonomy measurement system (SMAF): description and validation of an instrument for the measure of handicaps. Age and Ageing, 17, 293-302.

Lawton, M. P., Kleban, M., Moss, M., Rovin, M., Glicksman, A. (1989). Measuring caregiving appraisal, Fournal of Gerontology: Psychological Sciences, 57, 74-86.

Lewis, M., Hobday, J. V. and Hepburn, K. W. (2010). Internet-based program for dementia caregivers. American Fournal of Alzheimer's Disease and Other Dementias, 25, 674-679.

Mahoney, D., Tarlow, B. J. and Jones, R. N. (2003). Effects of an automated telephone support system on caregiver burden and anxiety: findings from the REACH for TLC intervention study. The Gerontologist, 43, 556567.

Marziali, E. and Donahue, P. (2006). Caring for others: internet video-conferencing group intervention for family caregivers of older adults with neurodegenerative diseases. The Gerontologist, 46, 398-403.

Marziali, E. and Garcia, L. J. (2011). Dementia caregivers' responses to 2 internet-based intervention programs. American fournal of Alzheimer's Disease and Other Dementias, $26,36-43$.

Magnusson, L., Hanson, E. and Nolan, M. (2005). The impact of information and communication technology on family carers of older people and professionals in Sweden. Aging and Society, 25, 693-713.

Parke, C. L., Cohen, L. H. and Murch, R. L. (1996). Assessment and prediction of stress-related growth. fournal of Personality, 64, 71-105.

Pearlin, L. I. and Schooler, C. (1978). The structure of coping, fournal of Health and Social Behavior, 19, 2-21.

Pettit, T., Livingstone, G., Manela, M., Kitchen, G., Katona, C. and Bowling, A. (2001). Validation and normative data of health status measures in older people: the Islington study, International fournal of Geriatric Psychiatry, 16, 1061-1070.

Posner, B. M., Jette, A. M., Smith, K. W. and Miller, D. R. (1993). Nutrition and health risks in the elderly: the nutrition screening initiative. American fournal of Public Health, 83, 972-978.

Powell, J., Chiu, T., and Eysenbach, G. (2008). A systematic review of networked technologies supporting carers of people with dementia. Fournal of Telemedicine and Telecare, 14, 154-156.

Radloff, L. S. (1977). The CES-D Scale: a self-report depression scale for research in the general population. Applied Psychological Measures, 1, 385-401.

Robison, J., Fortinsky, R., Kleppinger, A., Shugrue, N., \& Porter, M. (2009). A broader view of family caregiving: effects of caregiving and caregiver conditions on depressive symptoms, health, work, and social isolation. The fournals of Gerontology Series B: Psychological Sciences and Social Sciences, 64, 788-798.

Rosen, J., Mittal, V., Mulsant, B. H., Degenholtz, H., Castle, N. and Fox, D. (2003). Educating the families of nursing home residents: a pilot study using a computer-based system. Fournal of the American Medical Directors Association, 4, 128-134.

Russel, D. W., Peplau, L. T. and Cutrona, C. E. (1980). The revised UCLA loneliness scale: concurrent and discriminant validity evidence, Fournal of Personality and Social Psychology, 39, 472-480.

Schulz, R. and Beach, S. R. (1999). Caregiving as a risk factor for mortality: the caregiver health effects study. The Fournal of the American Medical Association, 282, 2215-2219.

Schulz, R. and Martire, L.M. (2004). Family caregiving of persons with dementia: prevalence, health effects, and support strategies. American fournal of Geriatric Psychiatry, $12,240-249$.

Schoenmakers, B., Buntinx, F. and Delepeleire, J. (2010). Factors determining the impact of care-giving on caregivers of elderly patients with dementia. A systematic literature review. Maturitas, 66, 191-200.

Sörensen, S., Pinquart, M., Habil, D. and Duberstein, P. (2002). How effective are interventions with caregivers? A updated meta-analysis. The Gerontologist, 42, 356-372.

Spielberger, C. D., Gorsuch, R. L., Lushene, R., Vagg, P. R. and Jacobs, G. A. (1985). Manual for the state-trait anxiety inventory (Form Y). Palo Alto, CA: Consulting Psychologists Press.

Steffen, A. M., McKibbin, C., Zeiss, A. M., Gallagher-Thompson, D. and Bandura, A. (2002). The revised scale for caregiving self-efficacy: reliability and validity studies. Fournal of Gerontology: Psychological Sciences, 57, 74-86.

Stoltz, P., Udén, G. and Willman, A. (2004). Support for family carers who care for an elderly person at home-a systematic literature review. Scandinavian fournal of Caring Sciences, 18, 111-119.

Tarlow, B. J., Wisniewski, S. R., Belle, S. H., Rubert, M., Ory, M. G. and Gallagher-Thompson, D. (2004). Positive aspects of caregiving: contributions of the REACH project to the development of new measures for Alzheimer's caregiving. Research on Aging, 26, 429-453.

Teri, L., Truax, P., Longsdon, R., Uomoto, J., Zarit, S. and Vitaliano, P. (1992). The revised memory and behaviour problems checklist. Psychology and Aging, 7, 622-631.

Torp, S., Hanson, E., Hauge, S., Ulstein, I. and Magnusson, L. (2008). A pilot study of how information and communication technology may contribute to health promotion among elderly spousal carers in Norway. Health and Social Care in the Community, 16, 75-85.

Van der Roest, H. G., Meiland, F. J. M., Jonker, C. and Dröes, R-M. (2010). User evaluation of the DEMentia-specific Digital Interactive Social Chart (DEM-DISC). A pilot study among informal carers on its impact, user friendliness and, usefulness. Aging and Mental Health, 14, 461-470.

Vernooij-Dassen, M. J., Felling, A. J., Brummelkamp, E., Dauzenberg, M. G., Van der Bos, G. A. and Grol, R. (1999). Assessment of caregiver's competence in dealing with the burden of caregiving for a dementia patient: a Short Sense of Competence Questionnaire (SSCQ) suitable for clinical practice. Fournal of the American Geriatrics Society, 47, 256-257. 
Vitaliano, P. P., Russo, J., Carr, J. E., Maiuro, R. D. and Becker, J. (1985) The ways of coping checklist: revision and psychometric properties. Multivariate Behavioural Research, 20, 3-26.

White, M. and Dorman, S. M. (2001). Receiving social support online: implications for health education. Health Education Research, 16, 693-707.

Wu, Y., Faucounau, V., de Rotrou, J., Riguet, M. and Rugaud, A-S. (2009). Intervention psychosociale aupres d'aidants familiaux de patients atteints de la maladie
d'Alzheimer et technologies de l'informaiton et de la communication: une revue de la literature / Information and communication technology interventions supporting carers of people with Alzheimer's Disease: a literature review. Psychologie and NeuroPsychiatrie du Vieillissement, 7, 185-192.

Zimit, G. D., Dahlem, N. W., Zimit, S. G. and Farley, G. K. (1988). The multidimensional scale of perceived social support. Fournal of Personality Assessment, 52, 30-41. 